\title{
Gerontology
}

Gerontology 2012;58:269-281

DOI: $\underline{10.1159 / 000329892}$
Received: September 3, 2010

Accepted: June 8, 2011

Published online: August 31, 2011

\section{Participatory and Persuasive Telehealth}

\author{
Duckki Lee $^{\mathrm{a}}$ Sumi Helal $^{\mathrm{a}}$ Steve Anton ${ }^{\mathrm{b}}$ Scott De Deugd ${ }^{\mathrm{C}}$ Andy Smith ${ }^{\mathrm{c}}$ \\ a Mobile and Pervasive Computing Laboratory, Computer and Information Science and Engineering Department \\ (CISE), and ${ }^{b}$ Department of Aging and Geriatric Research, University of Florida, Gainesville, Fla., and \\ 'Emerging Standards Division, IBM, Research Triangle Park, N.C., USA
}

\section{Key Words}

Connected health $\cdot$ Remote monitoring and intervention •

Service-oriented device architectures $\cdot$ Social network

applications in healthcare $\cdot$ Behavior alteration $\cdot$ Persuasive computing $\cdot$ Persuasive technology $\cdot$ Behavior change

theory $\cdot$ Behavior change model

\begin{abstract}
Technological advances in telehealth systems are primarily focused on sensing and monitoring. However, these systems are limited in that they only rely on sensors and medical devices to obtain vital signs. New research and development are urgently needed to offer more effective and meaningful interactions between patients, medical professionals and other individuals around the patients. Social networking with Web 2.0 technologies and methods can meet these demands, and help to develop a more complete view of the patient. Also many people, including the elderly, may be resistant to change, which can reduce the efficacy of telehealth systems. Persuasive technology and mechanisms are urgently needed to counter this resistance and promote healthy lifestyles. In this paper, we propose the participatory and persuasive telehealth system as a solution for these two limitations. By integrating connected health solutions with social networking and adding persuasive influence, we increase the chances for effective interventions and behavior alterations.

Copyright $\odot 2011$ S. Karger AG, Basel
\end{abstract}

\section{Introduction}

Advances in healthcare have led to longer life expectancy, resulting in an increasing proportion of the elderly population. By 2030, the number of US adults aged 65 years or older will more than double to about 71 million [1]. Moreover, about $80 \%$ of the elderly have one chronic condition, and $50 \%$ of the elderly population have two or more chronic conditions [1]. Chronic diseases disproportionately affect older adults and are associated with disability, diminished quality of life, and increased costs for healthcare and long-term care [1].

Many elderly people prefer independent living without caretakers, and industry and academia have put forth considerable effort to support independent living as well as provide cost-effective solutions to promote healthy aging [2-9].

Telehealth, or the delivery of health-related services and information via telecommunications technologies by a healthcare provider to persons that are some distance from the provider, is a cost-effective approach that could support independent living and facilitate telecare in general. However, telehealth is limited due to its dependence on sensors and medical devices to collect vital signs and its asymmetric focus on sensing and monitoring with little focus on affecting or altering behaviors. Innovation is urgently needed to offer a better and more effective telehealth system.

\section{KARGER}

Fax +41613061234

E-Mail karger@karger.ch

www.karger.com
(C) 2011 S. Karger AG, Basel

0304-324X/12/0583-0269\$38.00/0

Accessible online at:

www.karger.com/ger
Sumi Helal, PhD, Mobile and Pervasive Computing Laboratory

Computer and Information Science and Engineering Department (CISE)

University of Florida, Room 448, CSE Building

PO Box 116125, Gainesville, FL 32611 (USA)

Tel. +1 352392 6845, E-Mail helal@ cise.ufl.edu 
Considerable academic, industrial, standard organizational, and multinational efforts [2-11] have been put forth to improve telehealth systems, but they still miss valuable opportunities. Recent studies have attempted to leverage social networks to improve telehealth systems. Patientslikeme [10] is an online community built to connect patients who have similar diseases, and encourages the exchange of information, such as realworld treatments, symptom reports, etc., with each other. SenseFace [11], a sensor network overlay for social networks, is designed to deliver sensing information from the Body Sensor Network to a patient's social network.

Those works utilize social networks to share information and encourage collaboration. In our previous work [2], we tried to extend patient monitoring by adding a 'network effect', which is the collection of insights and views of people close to the patient, by incorporating a patient's social network to develop a more complete view of that patient. In our recent work [3], we attempted to increase the effectiveness of telehealth by adding persuasive influence using the action-based behavior model, developed by the University of Florida, which is designed to help telemetric researchers understand and apply technological intervention channels.

The combination of social networks and persuasiveness could provide several benefits. Participation of people within the social network of the patient can enhance the persuasive influence of behavior modification. Additionally, we can utilize people close to the patient to assist in overcoming barriers and problems that the patient may encounter. Furthermore, through the leveraging of social networks, people can cooperate to create a synergistic persuasive power so that the persuasive power can be further increased.

In this paper, we propose a participatory and persuasive telehealth system as a solution for the limitations of traditional telehealth systems. By integrating connected health solutions with social networking, as well as incorporating persuasive influences, more effective interventions and behavior alterations can be delivered.

To date, this unique and unprecedented opportunity, which is the synergistic combination of social networks and persuasive power to improve the efficacy of telehealth system, has not been explored. We would like to utilize this missed opportunity, through leveraging social networks and persuasiveness. We propose a framework that enables people in a patient's network to participate in the care of the patient to get a better view of the patient's overall well-being. Our proposed framework also adds persuasive power to increase the efficacy of telehealth systems in general.

In the following sections, we will give an overview of the existing telehealth systems and discuss their limitations. Furthermore, we will introduce enabling technologies and frameworks for participatory/persuasive telehealth with exemplifying scenarios and an architecture that can utilize such frameworks along with the actionbased behavior model we introduced in 2010 [3]. Additionally, we will provide implementation details and discuss future research.

\section{Present Telehealth Systems and Limitations}

Telehealth solutions have increased in prevalence and improved the quality of care by enabling the patient to take readings and measurements in the comfort of their own home. Personal medical devices are broadly available, affordable, and their accuracy of measurement is improving. Thanks to emerging standards such as the Continua Health Alliance [5], building highly interoperable telehealth systems using personal medical devices is becoming a reality and will hopefully lead to improved quality of care. In addition to standards adoption, there have been joint efforts between industry and academia on middleware enablers for telehealth systems. For example, STEPSTONE [4] is a joint project between IBM and the University of Florida to create technology that would enable a scalable, 'friction-free' integration of device-based healthcare solutions into enterprise systems using a service-oriented device architecture [12].

The Center for Connected Health [6], a division of Partners HealthCare in Boston, develops innovative and effective solutions for delivering quality patient care outside of the traditional medical setting. Two representative efforts for health record interoperation are Microsoft Healthvault [7] and Google Health [8]. Through these two Web portals (services), patients can easily manage their health records and share them with medical professionals. In Europe, the European Union funds programs such as the Ambient Assistive Living [9] to enhance the quality of life for older people, strengthening the industrial base in Europe through the use of information and communication technologies.

Many present telehealth systems, however, do not perform as effectively as would be expected. One important resource that telehealth systems miss, and one that may be critical to improve health monitoring, are the networks of people that are a part of everyone's personal and 
Fig. 1. Framework for participatory telehealth.

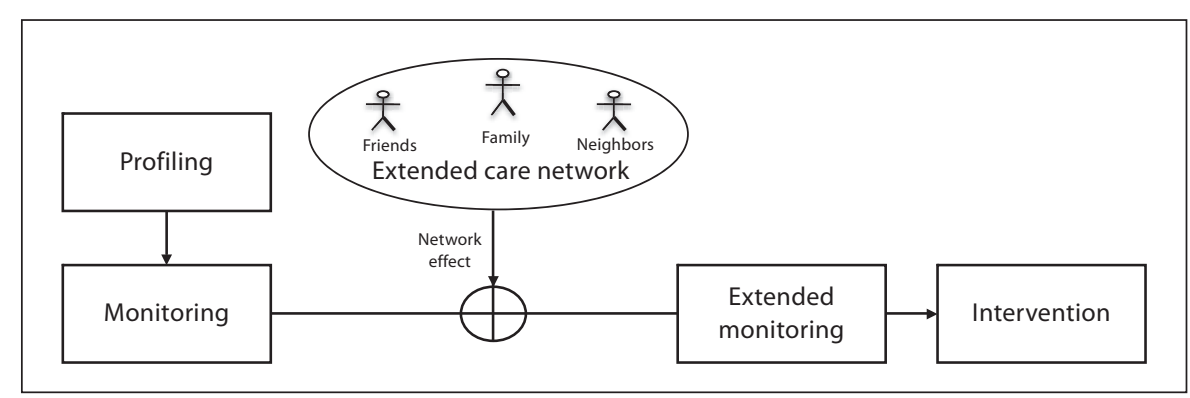

professional lives. People do not live in a vacuum, but rather are immersed in a variety of social networks, including family, friends, and neighbors. The knowledge contained within these networks can be used to augment the monitoring and contextualization of patients' lives. However, social networks are underutilized in telehealth systems. These networks often extend care to patients, thus making them an important resource in the overall care of a patient. By utilizing extended care networks, valuable information can be attained beyond vital signs, enabling a more complete view of the patient's status and well-being [2]. An effective telehealth system should create an environment in which those close to a patient would naturally participate in his/her monitoring.

Another problem with existing telehealth systems is their lack of support for persuasion. Many telehealth systems are asymmetric - they mostly provide capabilities to monitor and collect medical data, but offer very limited means to affect and change behavior. While sensing and monitoring are crucial components of a telehealth system, we believe that intervention and behavior alteration for affecting changes are equally important. Many people, especially the elderly, are resistant to change. Such resistance diminishes the impact factor of telehealth systems. Research and supportive technology for intervention and behavior alteration are urgently needed to counter this resistance to change and promote healthy lifestyles. Simple and naïve approaches for intervention, such as directly telling people what they need to do, have not been overtly successful. Thus, there is a need for a more advanced system. What we really need is persuasive power to make intervention and behavior alteration more effective [3].

\section{Participatory Telehealth}

In this section, we present our proposed framework and supportive scenarios for persuasive telehealth and its enabling technologies.

Participatory and Persuasive Telehealth

\section{Framework for Participatory Telehealth}

The participatory telehealth system develops an ecosystem in which people close to a patient would naturally participate in the monitoring of, and delivering care to, the patient.

We propose the framework shown in figure 1, which combines the typical telehealth framework with social networks to provide additional context beyond physical readings by adding the network effect from the extended care network [2]. This framework allows for the creation of a more comprehensive view of the user's overall health through participation of the patient's social networks [13].

In the profiling stage, any predefined or existing information, such as age, gender, medical history, preferences, etc., related to the user are collected. Vital signs are measured through sensors and medical devices in the monitoring stage. The 'network effect' refers to the collection of insights and data from the various perspectives of the extended care network, providing valuable information about the user, which is then added with vital signs in the extended monitoring stage. Monitoring of the user and user's activities can be extended by adding the network effect from the extended care network. Information gathered during this stage is then utilized in the intervention stage.

\section{Enabling Technologies for Participatory Telehealth}

A key technology that enables the participatory telehealth system is Web 2.0. Broadly speaking, Web 2.0 technologies enable individuals to produce and assemble content in new and unique ways. For example, blogs and Twitter make it possible for anyone to author and publish an article that is instantly accessible to a broad audience. Furthermore, Web 2.0 enables sites, such as iGoogle, to allow information to be collected, organized, and presented in a manner tailored specifically to that individual's needs and preferences using a technology called 'gadgets'. A gadget is a portable Web application that can be embedded in almost any Web page. 
Web 2.0 offers a different user experience than the typical 'patient portal'. Rather than requiring the user to navigate to a specific site, healthcare providers can offer their content via a gadget. This allows the healthcare provider to deliver content directly to the patient's choice of information portals while still maintaining the integrity of the source.

While content generation is the first step of gadget integration with personalized information portals, user interaction is also possible with gadgets. For example, consider a patient being treated for diabetes whose doctor has put them on a strict diet. Using Web 2.0 technology, the healthcare provider can offer a 'calorie counter' gadget that the patient can use to record their meals. This information can be used to update their health record, giving his/her doctor a continuous view of the patient's progress.

\section{Illustrative Scenarios}

Research work in participatory telehealth is often based upon common scenarios such as that of Charley, an aging patient with a series of ongoing health events that require his healthcare team to quickly adjust. Charley, an independently living senior, is managing his diabetes and related conditions through a tailored care plan with the help of his telehealth clinician, Josie. Charley's blood glucose level, weight, blood pressure, and daily activity information is automatically collected and sent to his healthcare provider's monitoring system, all configured according to a schedule and plan recommended by Charley's primary care physician [14]. In addition to the critical sensing and monitoring provided by telehealth systems, the collective and trusted experience of a patient like Charley's social network can be harnessed to extend and empower the concepts of participatory telehealth.

Josie monitors data collected from Charley on a daily basis through her Web browser. Because Charley's needs are constantly changing, new devices, care plan adjustments, and device settings are provided to him at home, so Josie frequently needs to add and remove different 'views' of Charley's data. Fortunately, this is a straightforward process because her 'patient management' page is a collection of gadgets, each with a specialized visual representation of the information collected by the remote monitoring service. For example, when Charley first received his blood pressure device, Josie simply added the 'blood pressure readings' gadget to her 'patient management' Web page and subscribed to a customized view of activities for Charley. Using these same mechanisms Josie is able to update these views with new gadgets her company provides, and she can also add third-party content that may be needed for the care of her patients, such as first responder profiles, FDA interaction warnings, CDC alerts [15], or care plan updates from equipment or primary care providers.

In addition to the physical readings, broader context about Charley beyond basic profiling and monitoring has proven to be increasingly valuable and accessible. These same Web 2.0 mechanisms also allow Charley and his extended care networks to leverage their existing online identities and relationships, checking in with Josie's health services system through custom gadgets shared via social networks and other Web 2.0 applications. This includes the ability to track dietary changes and daily questionnaires, as well as mood or behavioral changes reported from various perspectives of his extended care network.

Web 2.0-based social tools provide basic mechanisms to share profile information and authorize sharing of data, as well as to loosely coupled applications around a gadget model. With an extensive means to share both information and context with Charley and his extended care network of friends and family, care providers can establish a constant virtual connection to the important relationships around Charley, supporting his personal well-being as well as the delivery of his care.

\section{Persuasive Telehealth}

In this section, we present our proposed framework and illustrative scenario and its enabling technologies.

\section{Framework for Persuasive Telehealth}

When transportation is limited, the telephone is a convenient and inexpensive method for delivering nursing interventions and providing follow-up support. Traditional telehealth systems take an approach in which progressive steps begin with the development of a user profile, followed by monitoring based on that profile. Monitored vital data leads to analysis, followed by observation from medical professionals. Based on their observations, a nurse may perform a house call in the hope of motivating the user to change behaviors. Then, the nurse performs a follow-up call to record the results or suggest new strategies based on the user's response. However, during this process there could be communication errors, loss of information, and convergence could be slow due to the human-based model. This simple approach is also often ineffective in encouraging the user to change their behavior. Different intervention and convergence approaches 
Fig. 2. Framework for persuasive tele-

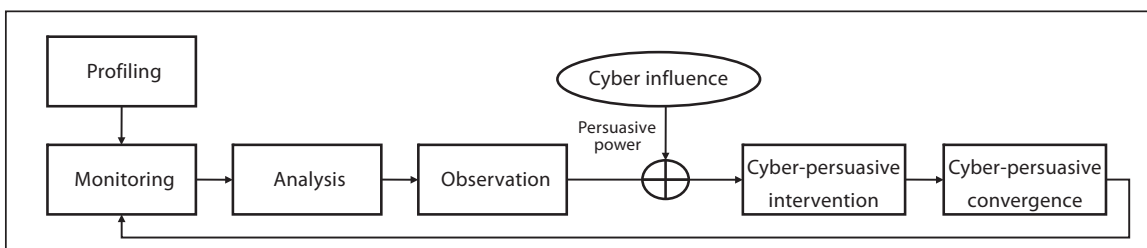
health.

are necessary to improve upon the traditional telehealth framework.

We propose the framework for persuasive telehealth as shown in figure 2, which combines the traditional telehealth framework with cyber influence to provide cyberpersuasive intervention and cyber-persuasive convergence by adding persuasive power [3]. In this framework we explicitly define the necessary steps and processes, emphasizing the intervention step in preparation for presenting our action-based behavior model in the next section. The framework consists of 6 steps, or stages, as shown in figure 2. We next describe each stage in greater detail.

Profiling. In this stage, user information and domainspecific knowledge are collected. User information is any predefined or existing information related to the user such as age, gender, medical history, disabilities, chronic conditions, preference/interest, and numerous other parameters. Domain-specific knowledge gives guidelines for analysis, and without this stage the system would struggle to infer this information dynamically using error-prone machine learning techniques.

Monitoring. Data most pertinent to a specific domain is monitored through a sensory subsystem. This data could range from physical status (e.g. vital signs, level and type of activities, food consumption, etc.) to mental status (e.g. mood, feeling, etc.). Environmental monitoring and other non-user conditions could also be pertinent to the goal of the telehealth system.

Analysis. Domain-specific analysis is performed based on the gathered data from the monitoring stage, qualified by the personalized/customized information of the enduser. For example, daily caloric expenditure could be calculated from an analysis of activities over the day based on a patient's height and weight. In order to analyze raw sensed data, some level of domain knowledge is required.

Observation. Domain experts, such as medical professionals, diagnose the current user health status and identify behavior and areas of concerns via the analyzed data. Such observations should drive the specifics of what needs to be changed.

Participatory and Persuasive Telehealth
Cyber-Persuasive Intervention. Many behavior theories and models emphasize the importance of motivation and ability in successful interventions [16-26]. In his behavior model, Fogg $[27,28]$ states that three factors, consisting of motivation, ability, and trigger, are necessary for behavior change. In our action-based behavior model, we utilize Fogg's work in the intervention process along with other popular persuasion approaches, such as goal setting and rewarding.

Cyber-Persuasive Convergence. In this stage, outcome assessment is performed and a determination is made as to whether any behavior change has actually materialized. Convergence towards the desired goals of the telehealth system will be measured by the change of the user behavior and status over time. Based on assessment outcomes, adaptation rules are applied in an attempt to stimulate convergence.

\section{Enabling Technologies for Persuasive Telehealth}

Successful implementation of persuasive telehealth would rely on an effective technology to achieve persuasion. We review existing technologies and discuss their suitability for persuasion.

Neither the more traditional house call, nor the more modern automated voice response are adequate for persuasion and intervention. There are not enough healthcare professionals to make house calls, and if they do, there are no guarantees the user is available at the time of call or that the user will be persuaded to alter their behavior. Automated voice response has always been confusing and often demands too much patience on the part of users.

Web portals are a pull technology that requires the user's attention and initiative to use. They can convey much more accurate and personalized data than traditional presentations of patient information, but they pose challenges to usability, especially for those who do not or cannot use computers (e.g. those with certain disabilities or older adults who may be reluctant). Mobile messaging such as SMS could be used as a technology channel for persuasion, but again messages can be ignored, especial- 
Fig. 3. Domestic robots.

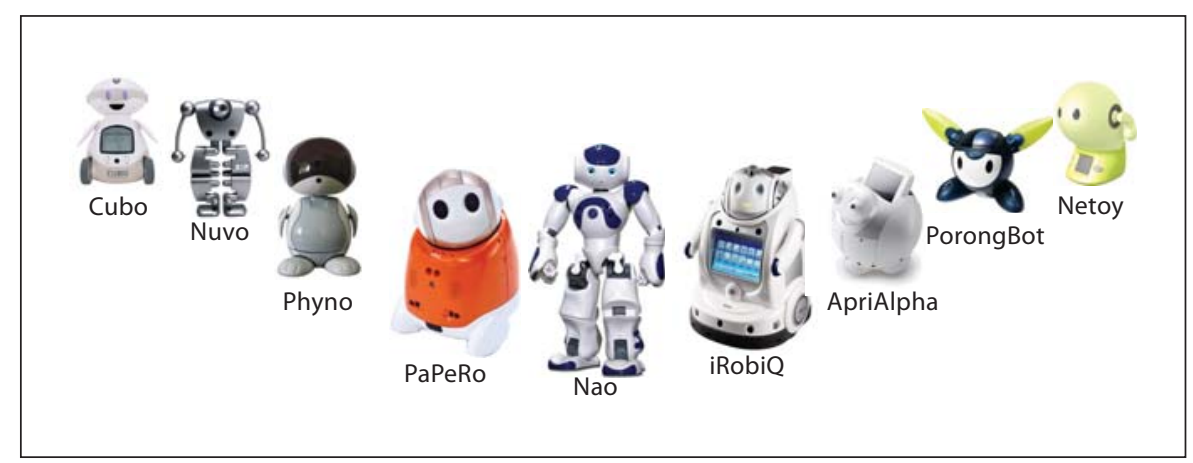

ly if the user becomes overwhelmed with the management of these messages (e.g. too much reading, deleting old messages, etc.).

Domestic robots (fig. 3) are a new technology that we have recently evaluated and consider to be a promising technology for persuasive telehealth. A recent survey of domestic robots can be found in Lee et al. [29]. Let us first describe the unique features of domestic robots in comparison to other devices. One reason some technologies are difficult to use in persuasive telehealth systems is because they require the user to spend effort learning and becoming familiarized with the technologies. Domestic robots are easier to use through their natural human-like communication, which can provide a pleasant experience for the user. Their friendliness can create an emotional bond [30,31], which may help the users, such as the elderly, feel more comfortable using them. Domestic robots are in fact effective informers, educators, reminders, and even readers of the user's feelings and thoughts, which are hard to detect using other devices.

Social networks can be an effective tool for setting appropriate goals, and can help find solutions for problems that may arise in pursuit of that goal. There will likely be a great diversity of problems, requiring expertise across a range of fields to effectively address them. Social networks may be the cheapest way to bring together experts, such as doctors, psychologists, and nutritionists, with the friends and family of patients. If we utilize this social network effect well, social networks could be a powerfully persuasive tool. The domestic robot can interact with this social network, and then act as an abstraction of the social network to the user.

\section{Illustrative Scenario}

In the following scenario, we demonstrate how persuasive telehealth works and how it differs from present telehealth systems.
Let us revisit the patient Charley. Charley is an elderly patient who has been managing diabetes. He joined a telehealth program and is therefore no longer required to visit a hospital for regular checkups. Charley has been provided devices to measure his blood pressure and glucose level. The telehealth system automatically gathers the data and communicates it in a visual format to the telehealth clinician, Josie, who uses the data to monitor Charley's health condition and to contact him about any abnormal trends. His doctor, William, orders Charley to follow a strict diet designed by a telehealth nutritionist to keep his glucose levels within a healthy range. Charley considers this diabetic diet overly restrictive and believes nonadherence will be of little consequence. He does not know precisely how to follow the diet, finding the instructions vague and complicated.

Josie informs Charley of a persuasive telehealth trial study, and he decides to participate. The persuasive telehealth trial still utilizes his monitoring devices, but increases Charley's understanding of his condition by providing him with medical data, and interactive means to interpret and understand it. The advice of his care providers begins to make sense as Charley starts to understand the nature of his condition and how his decisions affect it. Along with his increased understanding, Charley becomes more aware of the risks of nonadherence and of the benefits in following his care provider's advice. As Charley's awareness of his situation improves, he is more motivated to follow the diet and achieve the goals set by his doctor. With Charley's participation, the telehealth system's continual feedback becomes a more effective tool in helping him manage his diabetes. When Charley's vitals are off or he strays from his diet and medications, the system reminds him and his support network of friends and family. With active participation from Charley, the telehealth study not only gathers information on the effectiveness of treatment, but also measures the ex- 
Fig. 4. Architecture for participatory and persuasive telehealth.

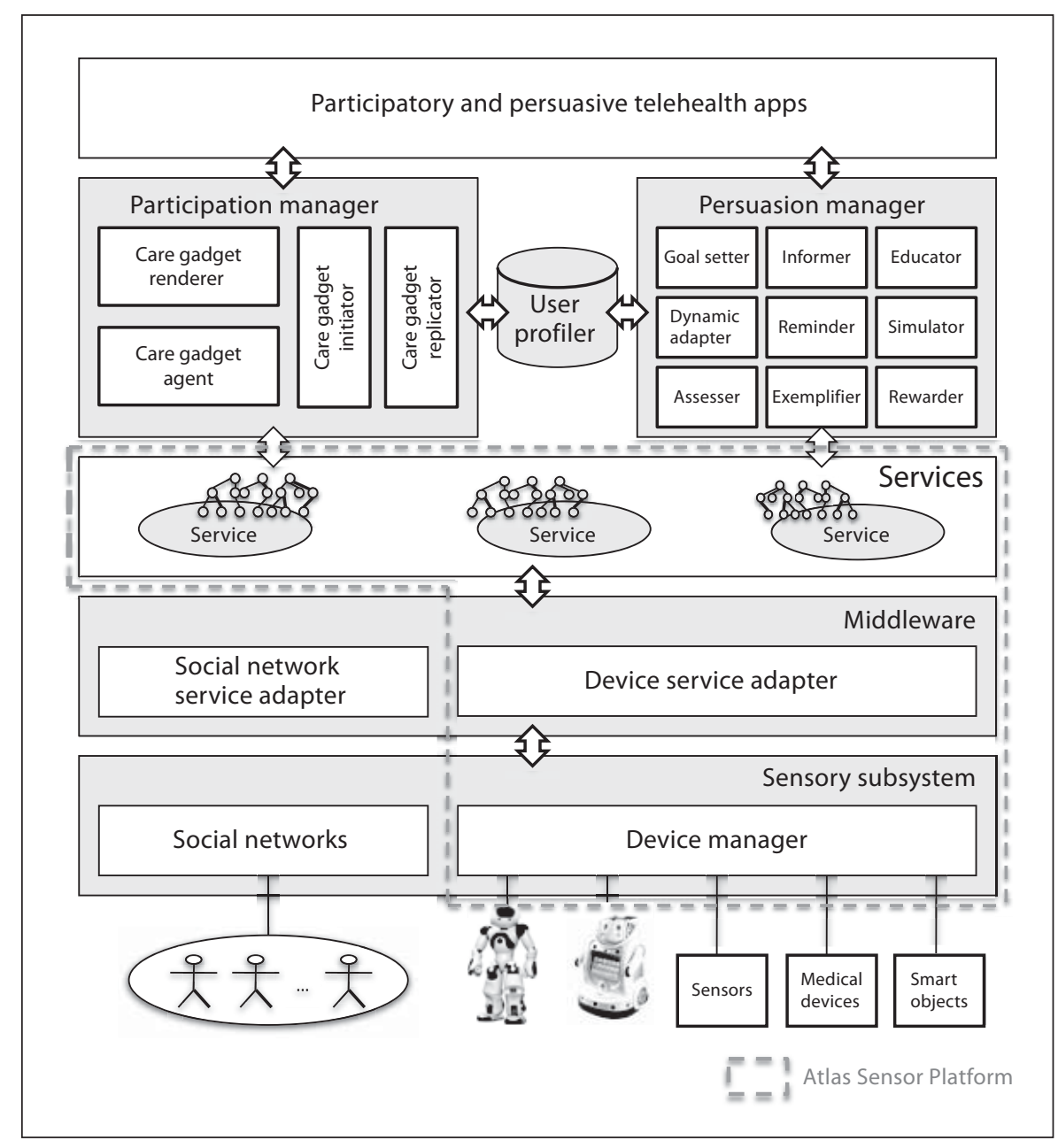

tent and value of patient participation. Information and analyses gathered from persuasive telehealth systems can be shared with family and friends in Charley's extended care network, and with health insurance companies looking for ways to incentivize better outcomes and lower costs.

\section{Architecture for Participatory and Persuasive Telehealth}

In this section, we describe the architecture for a participatory and persuasive telehealth system that utilizes the promising technologies and the framework described in the previous sections ('Participatory telehealth' and 'Persuasive telehealth'). As shown in figure 4, the architecture is designed to provide the means to the people around the patient to participate in (and the creation of)

Participatory and Persuasive Telehealth a persuasive influence to affect change toward a better and more effective telehealth system.

Starting from the bottom layer, the sensory subsystem consists of the social networks and the device manager. The social networks provide the means to expand and enhance the patient's extended care network. They are utilized by engaging people around the user such as family, friends, and medical professionals, encouraging effective and meaningful interactions to assist in setting appropriate care goals - either individually or collaboratively. The device manager controls several devices, including medical devices, smart objects, and domestic robots. The middleware layer contains the social network service adapter and the device service adapter. The social network service adapter is responsible for maintaining the service-oriented architecture view of the contributions of social networks. The device service adapter converts each sensor and device into services to provide the 
service-oriented device architecture [12]. The services layer provides mechanisms for discovery, configuration, and composition of services. It imposes a service view of the entire system and allows for the development of service-oriented applications.

The Atlas Platform $[32,33]$ is a service-oriented sensor platform developed at the University of Florida, and spans the device manager, the middleware layer for device services, and the services layer (dotted area in fig. 4). At the device manager level, a device description language (DDL) [34, 35] is used to generate an Atlas service bundle from the devices from the declarative specification.

The user profiler gathers user information including medical history, chronic conditions, preferences, interests, etc. This gathered information is utilized by the participation manager and the persuasion manager, which are key components to enable participatory and persuasive telehealth. The participation manager provides for the collection and consolidation of the informal care network input. The persuasion manager creates a persuasive influence to encourage changes in the patient's behavior.

The participation and persuasion applications layer consists of the execution environment that provides an application programming interface to access and control sensors, medical devices, smart objects, domestic robots, and social networks, as well as a tool to enable rapid and efficient development and deployment of the participation and persuasion telehealth applications. In the next sections, we will describe the details of the participation manager and the persuasion manager.

\section{Participation Manager}

The participation manager is responsible for the initiation, replication, and management of care gadgets. The care gadget initiator is used by an authorized care provider (Josie) to create a social network 'starter' (metaphorically similar to how a baker creates a starter for sourdough bread). The care gadget replicator is responsible for the proper spreading of the care gadget to the dynamic network of informal care providers (metaphorically, plays the same role of yeast in bread baking). The care gadget agent consolidates the contributions provided by the social network using aggregation and statistical techniques. It can also provide a spatiotemporal analysis of the stream of contributions, although we have not thoroughly investigated this possibility.

The following are the necessary protocols for the care gadget handshaking, replication, reporting and teardown.
Initiation

- The care gadget initiates communication with the participation manager by sending its XML representation file or an URL pointing to the file along with Charley's social network identity.

- When the participation manager receives the XML file, a care gadget initiator parses the XML file and checks compatibility through the 'require feature' in the XML file. If there are no parsing or compatibility problems, it accepts the request from the care gadget.

- Once the care gadget gains acceptance from the server, the handshaking procedure is done.

\section{Replication}

- The participation manager actively replicates the newly initiated care gadget and discovers Charley's current social network.

- For each member in Charley's extended care network, the care gadget replicator attempts to replicate the care gadget at the member whom Charley has authorized sharing.

- If accepted, each member handshakes with the participation manager.

\section{Reporting}

- Social members who receive and accept a replica of the care gadget enter several responses over time based on when they have time and observations to report.

- These responses initiate a reporting process by sending those responses to the participation manager.

- For each member in Charley's extended care network, the care widget agent in the participation manager processes the responses from each member.

\section{Tear-Down and Closing}

- When Josie recalls the care gadget, the care gadget initiator tries to close the care gadget.

- After closing, the care gadget agent generates statistics based on the responses.

\section{Persuasion Manager}

The persuasion manager is responsible for managing cyber influence, which is equivalent to pervasive space that has sensors and actuators. Through that space, cyber influence can provide the persuasive influence that may affect patient behavior. The persuasion manager provides persuasive influence actions that can affect changes in patient behaviors. The persuasion manager consists of persuasive influence actions, which are the informer, educator, reminder, exemplifier, simulator, assessor, goal 
Fig. 5. Action-based behavior model.

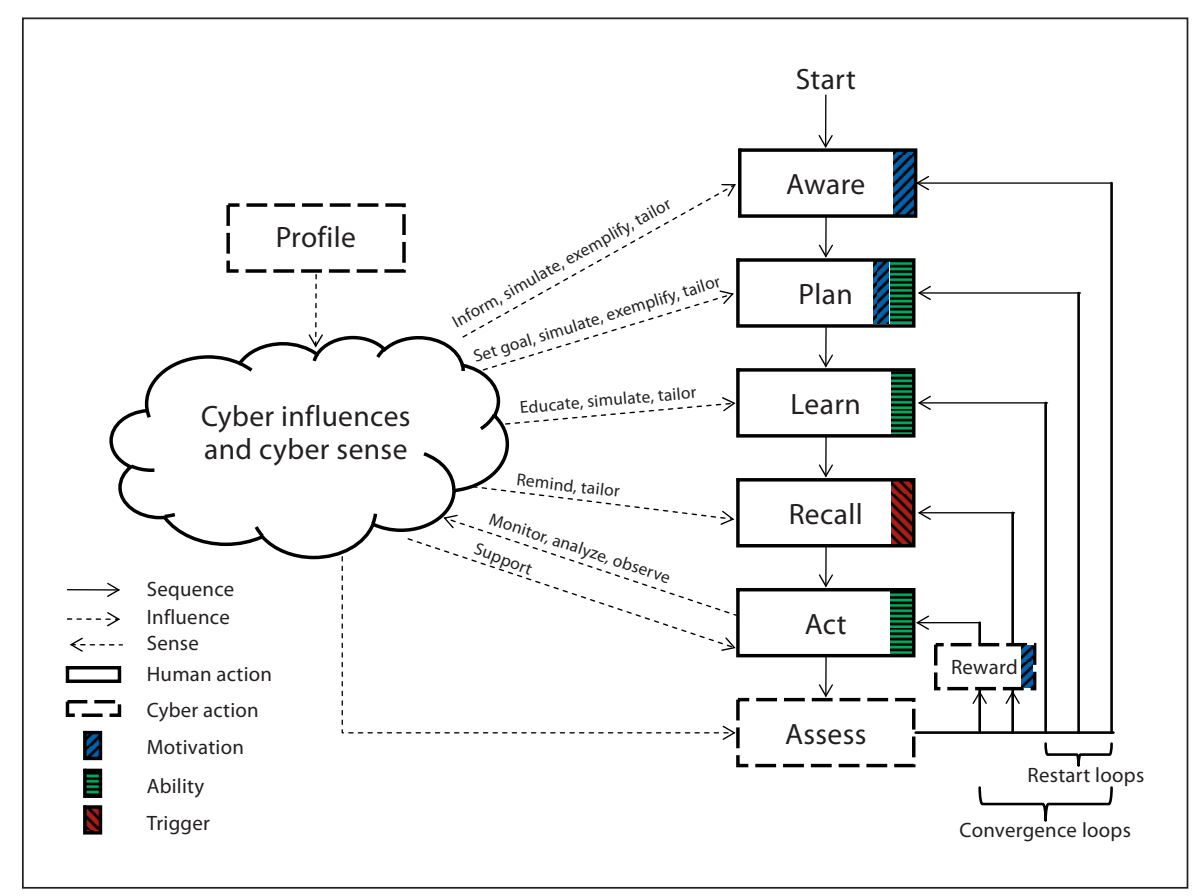

setter, rewarder, and dynamic adapter. Each action corresponds to the influence actions used by the cyber influence system in the action-based behavior model. These actions will be described in detail in the section 'Implementation of persuasive telehealth'.

\section{Action-Based Behavior Model}

Existing behavior change theories and models [16-26] explain how people change their behaviors. Each theory and model focuses on different factors such as self-efficacy; intention; and social, environmental, and personal factors. However, these models are not easily utilizable by health telematics researchers, including engineering and computer scientists. In this paper we describe a behavior change model that we believe is palatable and utilizable to the health telematics research community. The model is based on the collective knowledge we gained by studying social and behavioral science theories. Specifically, we propose an action-based behavior model that focuses on the intervention and convergence processes in the framework presented in the section 'Framework for persuasive telehealth'.

In this model, we first increase the user's awareness about their conditions by informing them about their current health status. This should increase their motivation by giving them the reasons for change. Next, we set goals. In this stage, we can utilize several types of goal setting: self-setting, assigned, participatory [36], and guided and group setting [37]. This process allows the users to understand the details of the goals and the benefits of achieving them. Then, we educate the users about how to achieve those goals. The next stage is reminding the users to act toward the goals. Even if the users are highly motivated and are capable to act and achieve the goals, they might simply forget. There are two manifestations to the reminding process. The first reminds the users to get them started acting towards the goal. The second is released gradually while achieving the goal to let the users know their progress toward the goals so they know where they stand. The final step is rewarding based on the achievement progress. Rewarding can be intrinsic (e.g. praise), extrinsic (e.g. gifts, credit, gift cards), or a virtual reward (e.g. virtual credit as well-practiced in games).

In our proposed action-based behavior model (figure 5), each step (represented as a rectangle) is an action and a state which can be easily understood and applied to the technological intervention channel. The model partitions the telehealth system into a cyber system and a set of user actions. The cyber component is further divided into cyber sensing and cyber influence to sense and influence exactly the set of actions prescribed by our model. The possible influence 'acts' that our model can prescribe are currently being finalized and include: inform, exem- 
Fig. 6. Participatory telehealth system architecture.

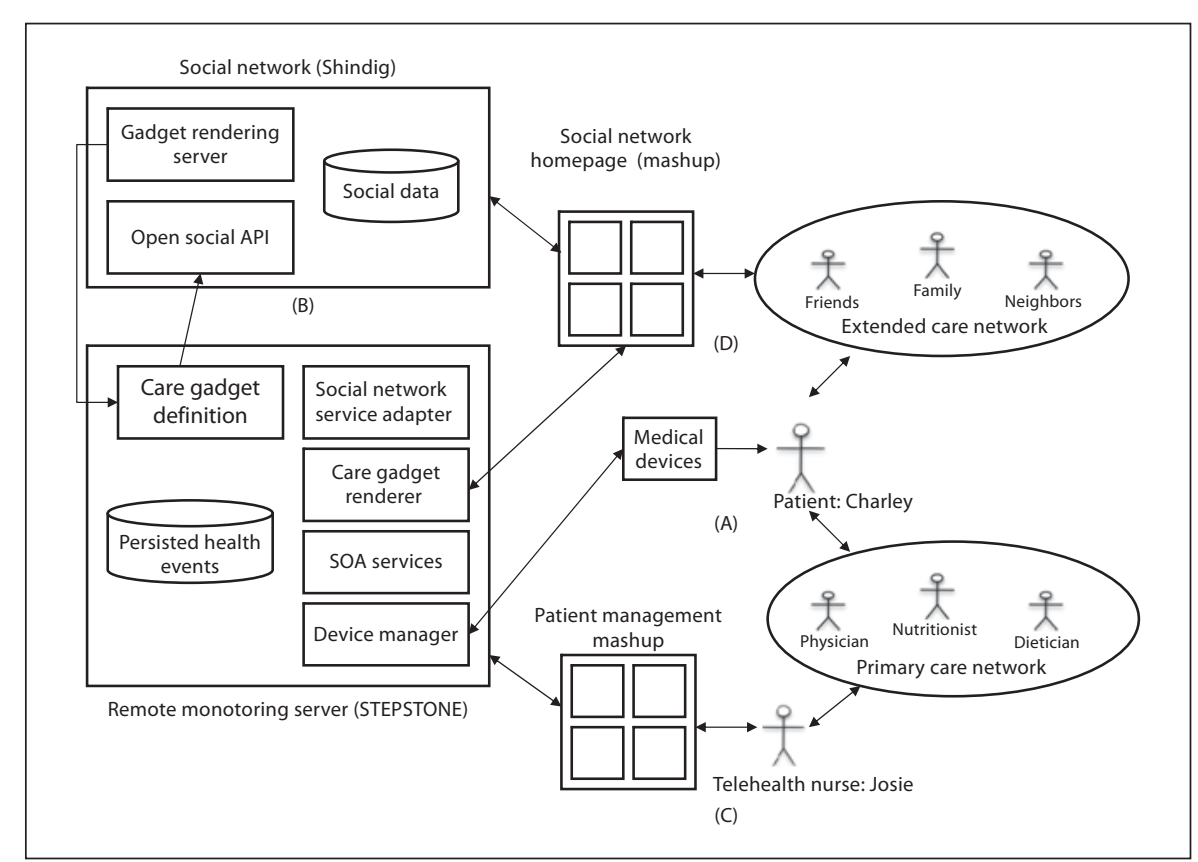

plify, simulate (e.g. play 'what if' scenarios) [38], tailor, educate, remind, monitor, morally support, analyze, and observe. These acts are shown above the influence/sense arrows in figure 5. Some influence acts may support different actions. For example, simulation increases self and goal awareness and provides effective education through a virtual experience.

In our model, cyber senses learn about the user's actions (or inactions), and in general senses any relevant vitals and status information. It also learns (initially) about the user's profile and preferences. Cyber influence, on the other hand, is where technological channels are used to manufacture controlled persuasion, within ethical guidelines [39]. Actions in the model consist of human actions (solid rectangles) and cyber actions (dotted rectangles). The intuition behind our model is that we designed it so that it guarantes progress of persuasion factors as the user (guided by the cyber system) steps through it. In fact, each human (and one cyber) action increases persuasion through one or more of its elements [27] (motivation, ability, and trigger). Figure 5 shows which element is contributed to by which action.

From 'start', a user goes through each step's action from 'aware' to 'act'. Cyber influence will affect and help the user to take each action. As the user works toward the goals, cyber senses will monitor, analyze, and observe the user's actions. After the initial rounds of acting, the 'assess' cyber action will evaluate the achievements of the goals and advise the user to either continue, or roll the user back to the appropriate action based on the achievement and deficit of each action. For positive achievements, the 'reward' cyber action may give intrinsic, extrinsic, or virtual rewards to reinforce the motivation.

\section{Implementation}

In this section, we describe implementations of the participatory and persuasive telehealth system. First, we briefly discuss implementations of a typical participatory telehealth system. We then include Web 2.0 technology integration with traditional remote monitoring services in support of the patients and their extended care networks. These are covered more extensively in 'Participatory medicine: leveraging social networks in telehealth solutions' [2] and 'A Web 2.0 model for patient-centered health informatics applications' [40]. Second, we provide guidelines for the integration of the telehealth system with the action-based behavior model [3].

\section{Implementation of Participatory Telehealth}

Participatory telehealth should provide an environment that the patient, the patient's extended care network, and primary care network can participate in for the care of the patient. Figure 6 demonstrates the implementation of such an environment, incorporating devic- 
Fig. 7. Persuasive telehealth system archi-

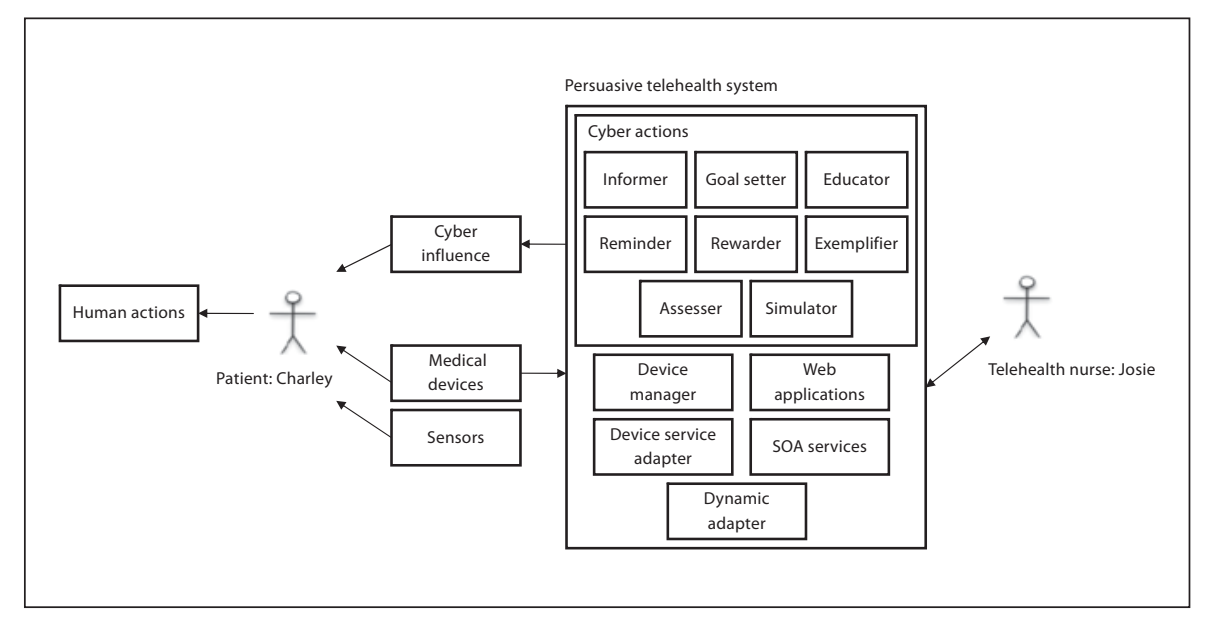

es, a remote monitoring server (RMS), and the social networks. We utilize STEPSTONE reference implementation $[4,12,41]$ and extend STEPSTONE to include social networking components from Shindig [42]. This combines medical devices with STEPSTONE to capture physical readings from the patient (part A in fig. 6). It also provides the gadget rendering server to visualize gadgets and the OpenSocial API [43] to support the retrieval of Charley's profile information, his relationships, and groups that he belongs to, as well as activities that he posted (part B in fig. 6). It demonstrates Josie is able to leverage a mashup displaying Charley's progress through a series of gadgets, which provide a portable interface into Charley's healthcare information (part C in fig. 6). It shows the users in Charley's network utilize a mashup to record their view of Charley's status through care gadgets (part D in fig. 6). Once this data is established, Josie can use her patient management mashup and the network effect of Charley's extended care network to gain new insights into Charley's status through the lens of his social network.

\section{Implementation of Persuasive Telehealth}

In the action-based behavior model, human actions (from 'aware' to 'act') indicate the required steps to change behavior. Each stage of human actions in the actionbased behavior model anticipates people's resistance, as well as other barriers that block or hinder moving to the next stage. Cyber influence actions help to counter such resistance and barriers, although detecting and measuring resistance is difficult. The key to successful implementation of persuasive telehealth systems is to increase the power of cyber influence enough to counter the resistance and barriers.

Participatory and Persuasive Telehealth
We described possible influence actions in the subsection 'Action-Based Behavior Model', but other influence actions are also possible. In general, these actions can be categorized into three different kinds of actions: (1) the main influence action, (2) the auxiliary influence action, and (3) the aid action. The main influence action is an action that directly affects humans to make a human action (e.g. inform, educate, remind, support). The auxiliary influence action is an action that indirectly encourages humans to take a human action (e.g. simulate, exemplify). The aid influence action (e.g. tailor) is an action that does not affect human action either directly or indirectly, but increases effectiveness of other actions. These actions can be used alone or in combination to achieve a synergistic effect.

Figure 7 shows the persuasive telehealth system architecture, which integrates the remote monitoring server with cyber actions. The system measures Charley's vital signs and gathers behavior information through medical devices and sensors. A telehealth nurse interacts with the persuasive telehealth system through Web applications to check Charley's status and generate cyber actions to influence him to take actions. The informer informs Charley of his medical professionals' observations regarding his conditions. The goal setter provides multiple methods to accomplish different types of goal settings. The educator gives specific strategies and steps toward goals. The reminder informs the patient to act toward goals at the appropriate times. The rewarder provides the means to offer intrinsic and virtual rewards. The exemplifier uses data mining techniques to find examples of others who have similar health status and goals, and strategies others have used to achieve their goals. The 
simulator requires interactions with the patient in order to get them to make choices, and provides a virtual experience according to the choice that the patient has made. The assessor performs outcome assessments of behavior changes and guides the user to either continue or re-step the appropriate actions based on achievement and deficits. The dynamic adapter provides mechanisms to dynamically change the system according to the adaption rules.

\section{Conclusions and Future Work}

This paper reviewed the limitations of present telehealth systems and promising technologies and frameworks that could enable the participatory and persuasive telehealth system. Web 2.0, social networking, domestic robots, persuasion technology, open source, and stan- dards are all ingredients for the next wave of innovation in telehealth sytems as we move towards the participatory and persuasive telehealth system framework.

We presented a set of scenarios and a technical approach demonstrating how social networks and persuasion technology can be leveraged to improve patient care through the 'network effect' and persuasive influence.

We propose the participatory and persuasive telehealth system as a solution for the limitations of present telehealth systems by integrating connected health solutions with social networking and adding persuasive influence to increase the effectiveness of interventions and behavior alterations.

For future research, we plan to deploy our proposed participatory and persuasive telehealth system in the Gator Tech Smart House [44], a real-world test-bed for participatory and persuasive telehealth field studies.

\section{References}

1 Healthy Aging: Improving and Extending Quality of Life Among Older Americans: At a Glance 2009. http://www.cdc.gov/chronicdisease/resources/publications/AAG/aging. htm.

2 Weitzel M, Smith A, Lee D, Deugd S, Helal S: Participatory medicine: leveraging social networks in telehealth solutions; in Mokhtari M, Khalil I, Bauchet J, Zhang D, Nugent C (eds): Ambient Assistive Health and Wellness Management in the Heart of the City. Heidelberg, Springer, 2009, vol 5597, pp $40-$ 47.

3 Lee D, Helal S, Johnson BD: An action-based behavior model for persuasive telehealth. Proceedings of the 8th International Conference on Smart Homes and Health Telematics (ICOST), Seoul, June 2010.

4 Helal A, Bose R, Chen C, Smith A, de Deugd S, Cook D: STEPSTONE: a SODA case study in personal tele-health management. J Ambient Intell Smart Environ, submitted.

5 The Continua Health Alliance. http://www. continuaalliance.org.

6 The Center for Connected Health. http:// www.connected-health.org.

7 Microsoft Health Vault. http://www.healthvault.com.

8 Google Health. https://health.google.com/ health.

9 Ambient Assisted Living Joint Programme. http://www.aal-europe.edu/.

10 Patientslikeme. http://www.patientslikeme. $\mathrm{com} /$.
11 Rahman A, El Saddik A, Gueaieb W: SenseFace: a sensor network overlay for social networks. IEEE International Instrumentation and Measurement Technology Conference, Singapore, 2009.

12 de Deugd S, Carroll R, Kelly KE, Millett B, Ricker J: SODA: service-oriented device Architecture. IEEE Pervasive Comput 2006;5: 94-97.

13 Fox S: Participatory Medicine: Text of My Speech at the Connected Health Symposium.http://e-patients.net/archives/2008/11/ participatory-medicine-text-of-my-speechat-the-connected-health-symposium.html.

14 A Smarter World for Charley. http://www. youtube.com/watch? $\mathrm{v}=$ QUEXdDxO37c1.

15 The Centers for Disease Control (CDC). http://www.cdc.gov/widgets.

16 Glanz K, Rimer BK, Lewis FM: Health Behavior and Health Education. Theory, Research and Practice. San Fransisco, Wiley \& Sons, 2002.

17 Becker MH: The health belief model and personal health behavior. Health Educ Monogr 1974;2:324-508.

18 Rosenstock I: Historical origins of the health belief model. Health Educ Monogr 1974;2: 328-335.

19 Prochaska JO, Velicer WF, Rossi JS, et al: Stages of change and decisional balance for 12 problem behaviors. Health Psychol 1994; 13:39-46.

20 Prochaska JO, DiClemente CC, Norcross JC: In search of how people change - applications to addictive behaviors. Am Psychol 1992;47:1102-1114.
21 Sheppard BH, Hartwick J, Warshaw PR: The theory of reasoned action: a meta-analysis of past research with recommendations for modifications and future research. J Consumer Res 1988;15:325-343.

22 Fishbein M: A theory of reasoned action: some applications and implications; in Howe H, Page M, Lincoln NB (eds): Nebraska Symposium on Motivation. Lincoln, University of Nebraska Press, 1980, pp 65-116.

23 Ajzen I: The Theory of Planned Behavior. Organ Behav Hum Decis Process 1991;50: 179-211.

24 Mearns J: Social learning theory; in Reis H, Sprecher S (eds): Encyclopedia of Human Relationships. Thousand Oaks, Sage, 2009, vol 3, pp 1537-1540.

25 Bandura A: Social cognitive theory: an agentive perspective. Annu Rev Psychol 2001;52: $1-26$.

26 Rotter JB: The Development and Applications of Social Learning Theory. Selected Papers. Englewood Cliffs, Prentice Hall, 1982.

27 Fogg BJ: A behavior model for persuasive design. Proceedings of the 4th International Conference on Persuasive Technology (PERSUASIVE), Claremont, 2009.

28 Fogg BJ: Persuasive Technology: Using Computers to Change What We Think and Do. San Francisco, Morgan Kaufmann Publishers, 2003.

-29 Lee D, Yamazaki T, Helal S: Robotic companions for smart space interactions. IEEE Pervasive Comput 2009;8:78-84. 
30 Kidd C, Breazeal C: Designing a sociable robot system for weight maintenance. IEEE Consumer Communications and Networking Conference, Las Vegas, 2006.

31 Sung J, Guo L, Grinter R, Christensen H: 'My Roomba Is Rambo': Intimate Home Appliances. Proc of Ubicomp. Springer, 2007, pp 145-162.

32 ATLAS Website. http://www.pervasa.com/.

33 King J, Bose R, Yang H, Pickles S, Helal A: Atlas - a service-oriented sensor platform. Proceedings of the First IEEE International Workshop on Practical Issues in Building Sensor Network Applications, Tampa, 2006.

34 DDL Webiste. http://www.icta.ufl.edu/atlas/ $\mathrm{ddl} /$.
35 Chen C, Helal A: Device Integration in SODA Using the Device Description Language. Ninth Annual International Symposium on Applications and the Internet, Seattle, 2009, pp 100-106.

36 Locke EA, Latham GP: Building a practically useful theory of goal setting and task motivation: a 35-year odyssey. Am Psychol 2002;57:705-717.

37 Shilts MK, Horowitz M, Townsend MS: Goal setting as a strategy for dietary and physical activity behavior change: a review of the literature. Am J Health Promot 2004;19:81-93.

38 King P, Tester J: The landscape of persuasive technologies. Commun ACM 1999;42:5158.
39 Berdichevsky D, Neunschwander E: Towards an ethics of persuasive technology. Commun ACM 1999;42:51-58.

40 Weitzel M, Smith A, de Deugd S, Yates R: A Web 2.0 model for patient-centered health informatics applications. Computer 2010;43: 43-50.

41 Open Health Tools Project. https://stepstone.projects.openhealthtools.org/.

42 The Apache Shindig Project. http://incubator.apache.org/shindig.

43 The Open Social Foundation. http://www. opensocial.org.

44 Helal S, Mann W, Zabadani H, King J, Kaddoura Y, Jensen E: The Gator Tech Smart House: a programmable pervasive space. IEEE Computer 2005;38:50-60. 